\title{
A REVIEW OF THE MEDICINAL LYCOPODIOPHYTA OF UKRAINE
}

\author{
Tetyana Dvirna ${ }^{1}$, Valentyna Minarchenko ${ }^{2}$, Iryna Tymchenko ${ }^{1}$ \\ ${ }^{1}$ Department of Systematics and Floristics of Vascular Plants, \\ M.G. Kholodny Institute of Botany, National Academy of Sciences of Ukraine \\ ${ }^{2}$ Department of Pharmacognosy and Botany, M.G. Kholodny Institute of Botany, \\ National Academy of Sciences of Ukraine
}

\begin{abstract}
The publication presents the results of complex research and analysis of medicinal plain-like of flora of Ukraine. Lycopodiophyta of Ukraine includes four families: Lycopodiaceae (8 species - Diphasiastrum alpinum (L.) Holub, D. complanatum (L.) Holub, D. isseleri (Rouy) Holub, D. tristachyum (Pursh) Holub, D. zeilleri (Rouy) Holub, Lycopodiella inundata (L.) Holub, Lycopodium annotinum L., L. clavatum L.), Huperziacaeae (1 - Huperzia selago (L.) Bernh. ex Schrank \& C. Mart.), Selaginellaceae (2 - Selaginella helvetica (L.) Spring., Selaginella selaginoides (L.) C. Mart.) and Isoëtaceae (1 - Isoëtes lacustris L.). They have different contents of biologically active substances and all of these species are medicinal or semi-medicinal. The paper presents the characteristics of their distribution in Ukraine, resource importance, population structure, environmental protection status in Ukraine and Europe, main active substances, and medicinal properties.
\end{abstract}

Keywords: medicinal Lycopodiophyta, resources significance, protection, Ukraine

\section{INTRODUCTION}

Nowadays, medicinal plants attract attention because they are among the main sources of medical and preventive means of non-traditional and scientific modern medicine (1-5).

One of the oldest and most interesting groups of plants is the representatives of Lycopodiophyta, which achieved their greatest development in the late Paleozoic. Currently it is represented by a relatively small number of genera and species, which account

\footnotetext{
Address for correspondence:

Tetyana Dvirna

M.G. Kholodny Institute of Botany

National Academy of Sciences of Ukraine

2 Tereshchenkivska St

01601 Kiev (Kyiv)

Ukraine

e-mail:dvirna_t@ukr.net
}

Received: April 8, 2019

Accepted: June 242019 for only $1 \%$ of the modern species diversity of vascular plants (6-8).

Extinct lycopods had an important role in the formation of coal $(6,9)$ while modern species have phytogenic, industrial, decorative and medicinal value (1-6,10-12).

Lycopods are able to form continuous carpets in the woods and participate in the formation of plant communities, which constitutes their phytogenic value. Spores of Lycopods are rich in fatty oils and aluminum, which is a reason for their use in pyrotechnics (for the manufacture of fireworks), metallurgy (for smoothness of surfaces at packed casting), in the manufacture of photos, where they are a source of green, blue and yellow colors. Plants are harvested for making bouquets and for various rituals, some species of Selaginella are indoor ornamental plants $(6,9,10,13)$.

The medicinal properties of the plants were noted by the ancient Romans, who considered them the best remedy for eliminating dullness of the skin (2). Nowadays, spores (lycopodium) are used as a 
powder (as a baby powder, for treatment of bedsores), and for pill coating, grass lycopsids are used as a diuretic, anti-rheumatic, anti-inflammatory, and anesthetic agent, in the treatment of mental illness and Alzheimer's disease; species of Huperzia are poisonous but used for the treatment of alcoholism and nicotinism $(1-3,5,11,14)$.

Lycopodiophyta of Ukraine includes four families: Lycopodiaceae (8 species - Diphasiastrum alpinum (L.) Holub, D. complanatum (L.) Holub, D. isseleri (Rouy) Holub, D. tristachyum (Pursh) Holub, D. zeilleri (Rouy) Holub, Lycopodiella inundata (L.) Holub, Lycopodium annotinum L., L. clavatum L.), Huperziacaeae (1 - Huperzia selago (L.) Bernh. ex Schrank \& C. Mart.), Selaginellaceae (2 - Selaginella helvetica (L.) Spring., Selaginella selaginoides (L.) C. Mart.) and Isoëtaceae (1 - Isoëtes lacustris L.) $(7,8)$ are medicinal or semi-medicinal.

Below we present a more detailed description of these species (Table 1).

\section{AIM AND METHODS}

The object of our study are the medicinal and semi-medicinal species of the Lycopodiophyta representatives of the flora of Ukraine. The basis of the work is the data obtained during the field research as a result of working on literary materials and maps, and herbarium collections of Ukraine gathered in the period 2010-2019.

\section{RESULTS AND DISCUSSION}

Lycopodiophyta species are of no economic value in Ukraine due to several reasons. The results of the study are presented in Table 1 in which the checklist of representatives of the studied department is given in alphabetical order. The name of each species is in Latin and English (9 species), presented are also family, distribution in Ukraine, resource value, population structure, conservation status in Ukraine and Europe, main active substances, and medicinal properties.

We analyzed the distribution of plant species in Ukraine and established that the majority $42 \%$ (5 species) - is known from several localities, mainly growing in the Carpathians and Polissya $(10,12,13,15,16)$.
The studied species of Lycopodiophyta have no resource values since most species ( $83 \%$ or 10 species) in the wild are not available.

The population structure of the species is unstable. However, $42 \%$ (5 species) are represented by several individuals, which is caused by a number of threats, but the main one is the anthropogenic factor (cutting, grazing, recreation, etc.) to which species are very sensitive $(10,13)$.

All species are listed in the Red Book of Ukraine and require conservation measures. Most of them $42 \%$ (5 species) - are vulnerable and only one species (Lycopodium clavatum) is regionally rare. L. clavatum and Huperzia selago are included in the European Red List, to the IUCN database with LC status, and to the European Red List of Medicinal Plants (13,17-19).

The pharmacological properties of the species emerge due to the chemical composition and the ratio of active ingredients. For medicinal purposes, spores and aerial parts of plants are used as raw material. The analysis of literary data enabled us to establish that model species that are taken as the basis for the research of pharmacological properties usually are L. clavatum and H. selago. It is caused by their chemical composition, which slightly differs from "other composition and ratio of components", while the properties, uses, and dosage forms are similar (1,20-24).

The main common active substances of Lycopodiophyta are alkaloids (lycopodine, clavatin, lycodine, etc.), flavonoids, triterpenoids, flavones, gupercine, carotenoids, fatty oils (spores) (1-3,5,6,11,20-38).

The spores of Lycopods contain about 50\% non-drying fatty oil and glycerides, stearic, oleic, arachidonic, palmitic and other acids, therefore, they are used as a powder for infants, for the treatment of eczema, adulthood, bedsores, wounds, burns $(1,2,4-6,11)$.

Spores and herbs are used in liver disease, respiratory tract diseases, and as diuretic, antirheumatic, anticonvulsant and anti-inflammatory, antibacterial and antifungal, antiviral and antiemetic agents, etc., as well as for the treatment of psychological disorders, Alzheimer's disease, alcoholism and nicotine $(1-5,11,38)$. 


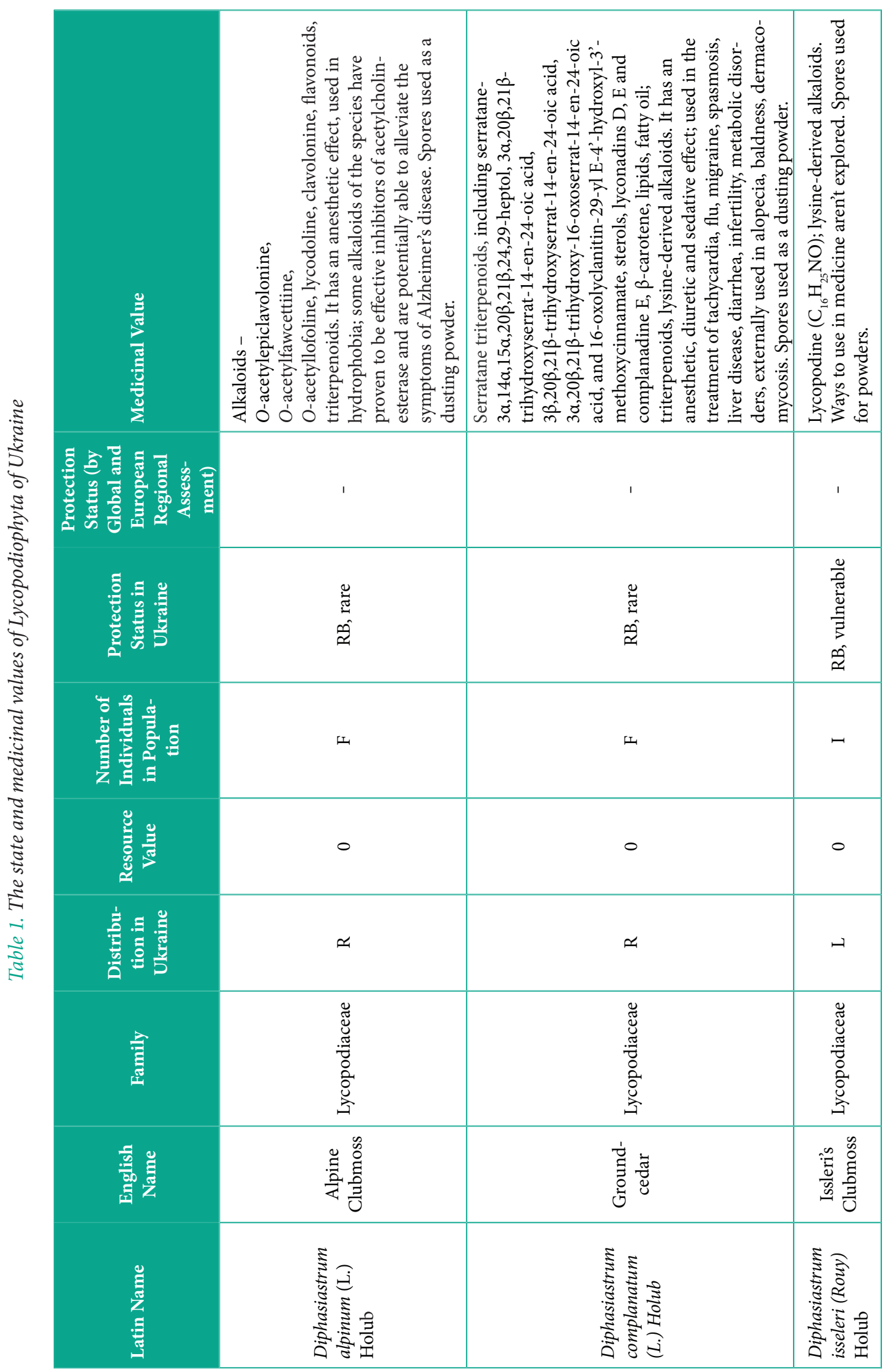

Scripta Scientifica Pharmaceutica, 2019;6(1):7-15 


\begin{tabular}{|c|c|c|}
\hline 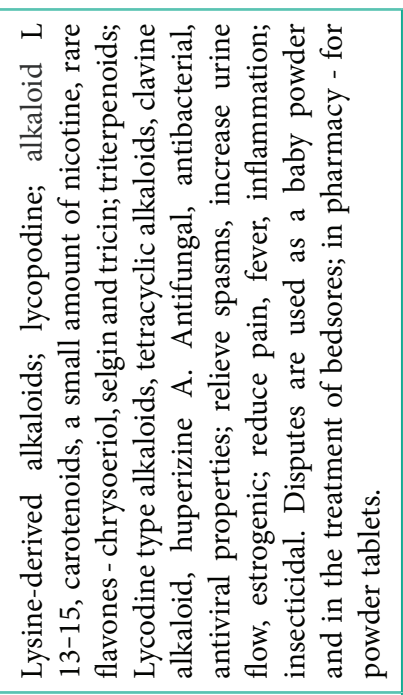 & 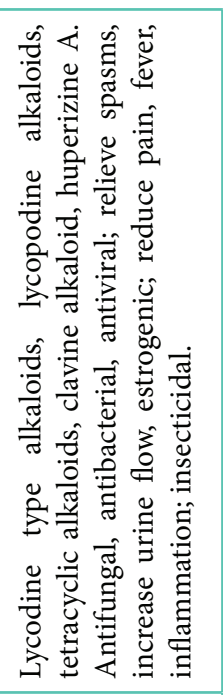 & 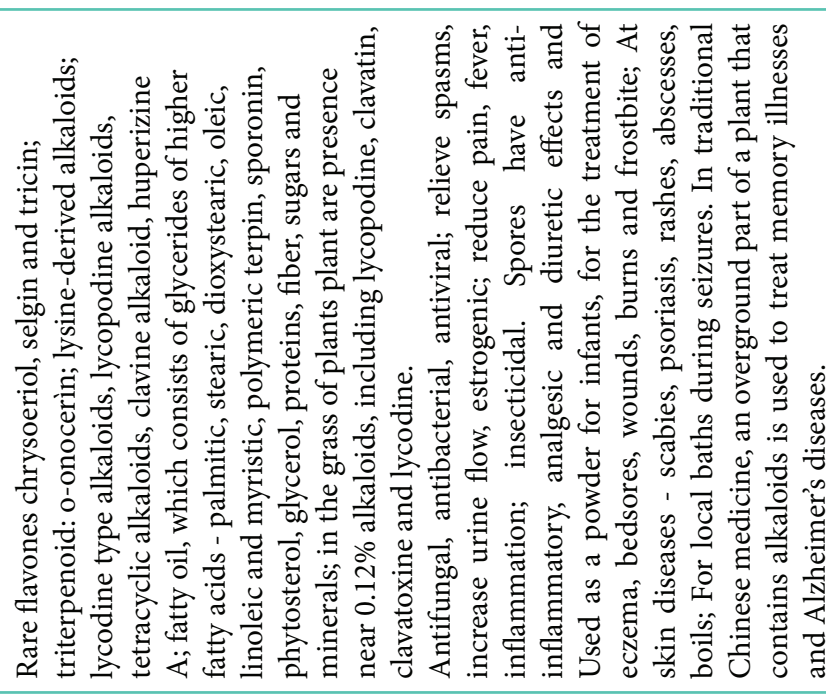 \\
\hline 1 & 1 & ' \\
\hline 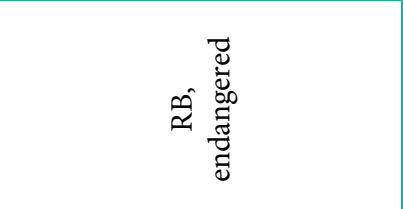 & 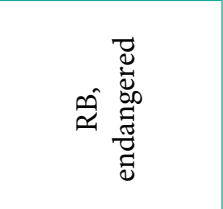 & 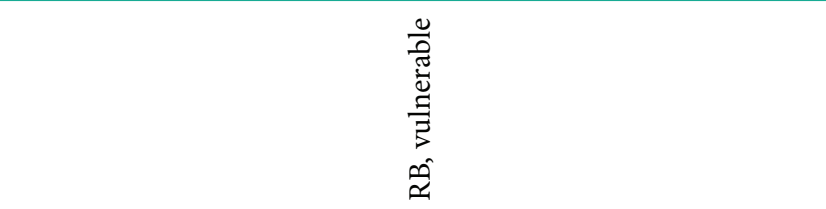 \\
\hline- & - & $z$ \\
\hline 0 & 0 & 0 \\
\hline لـ & ـ & $\Sigma$ \\
\hline 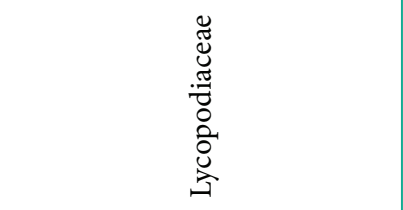 & 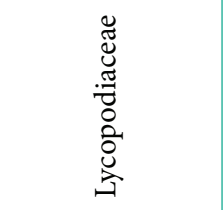 & 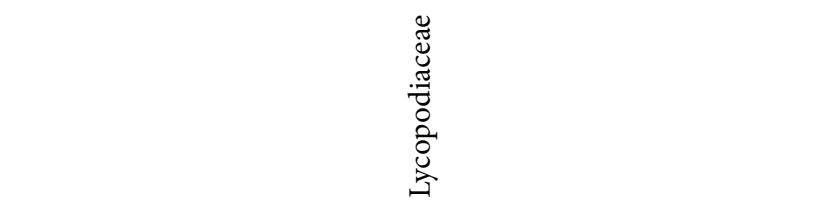 \\
\hline 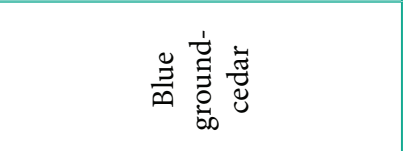 & 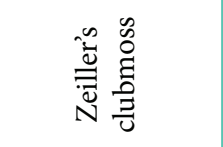 & 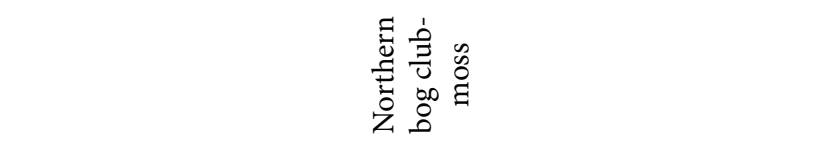 \\
\hline 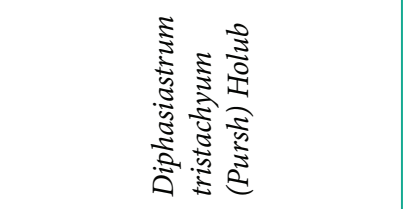 & 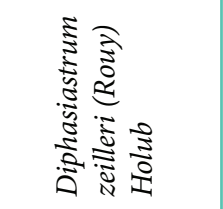 & 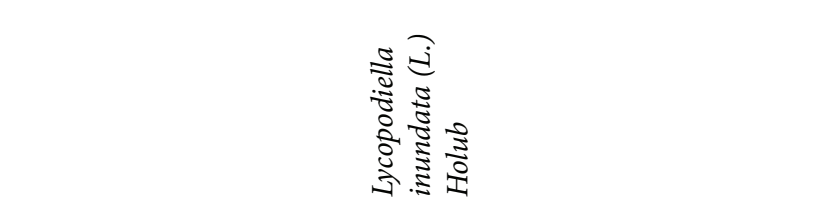 \\
\hline
\end{tabular}


Tetyana Dvirna, Valentyna Minarchenko, Iryna Tymchenko

\begin{tabular}{|c|c|}
\hline 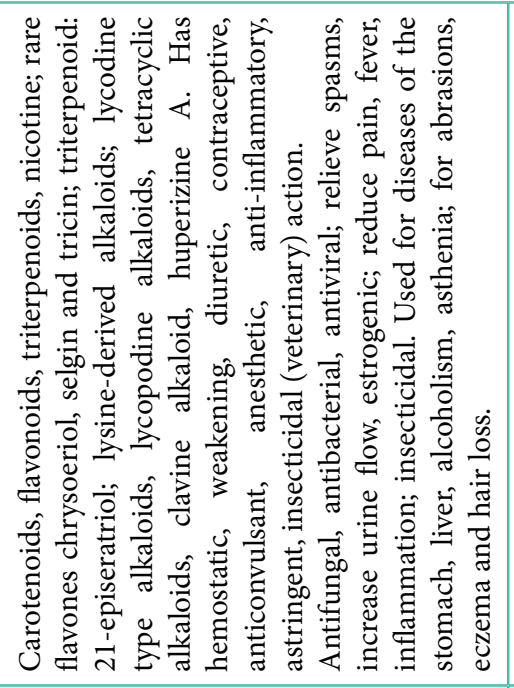 & 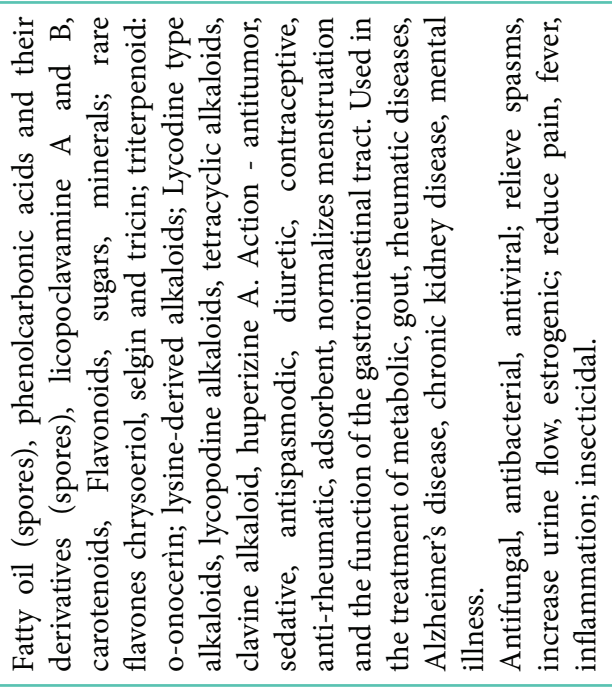 \\
\hline 1 & $\stackrel{\circlearrowleft}{\hookrightarrow}$ \\
\hline 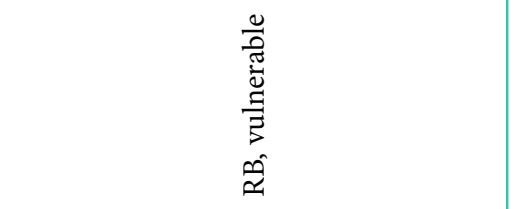 & حิ) \\
\hline $\mathrm{z}$ & $z$ \\
\hline 0 & $\Xi$ \\
\hline$\Sigma$ & $\Sigma$ \\
\hline 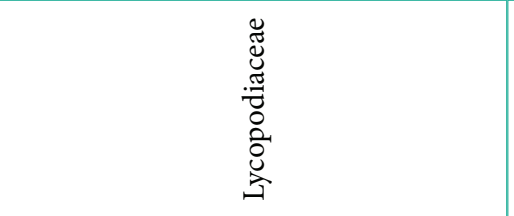 & 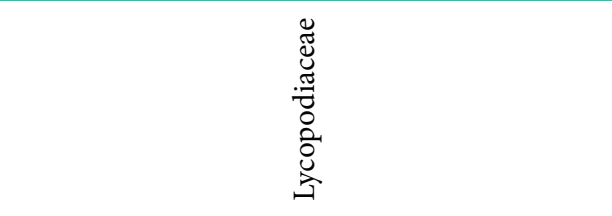 \\
\hline 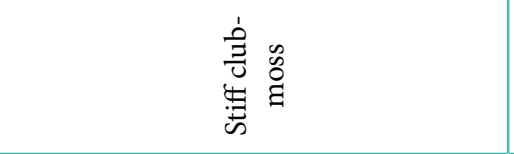 & 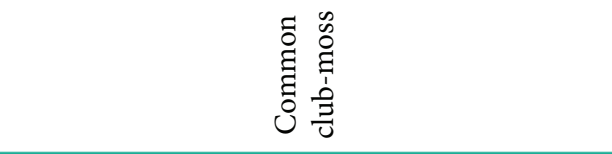 \\
\hline 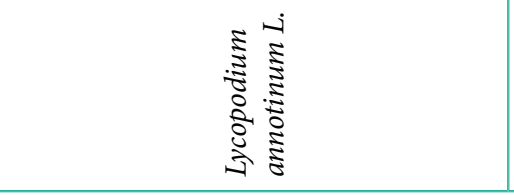 & 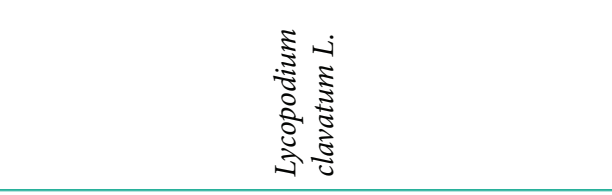 \\
\hline
\end{tabular}

Scripta Scientifica Pharmaceutica, 2019;6(1):7-15

Medical University of Varna 


\begin{tabular}{|c|c|c|c|}
\hline 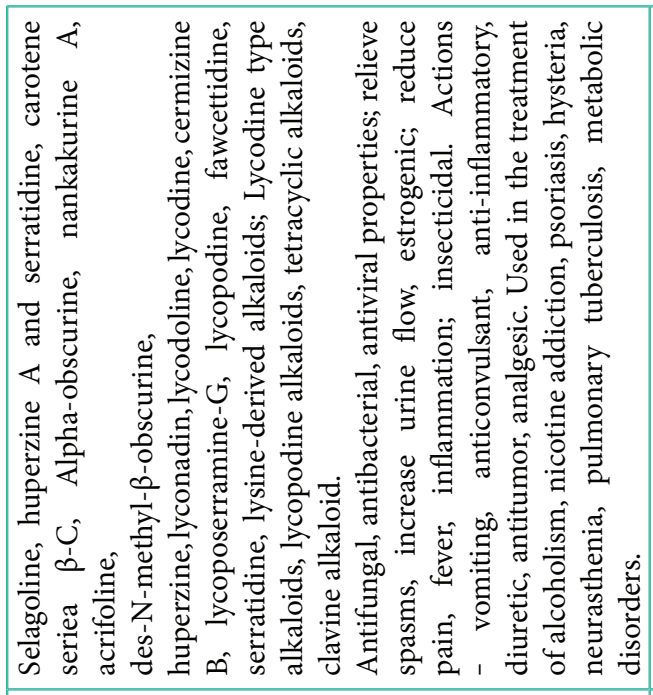 & 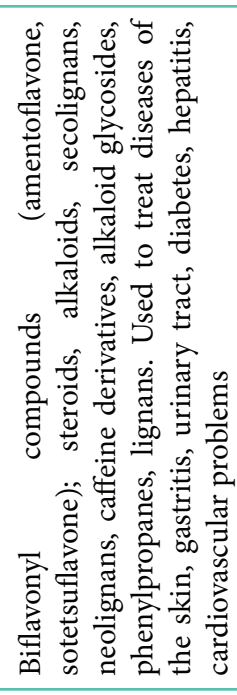 & 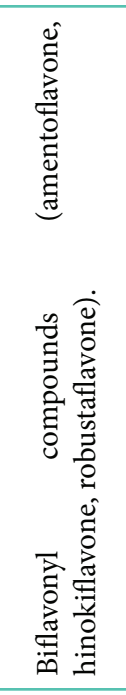 & 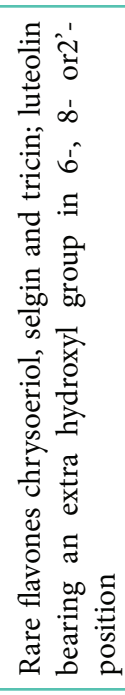 \\
\hline U & & 1 & 1 \\
\hline 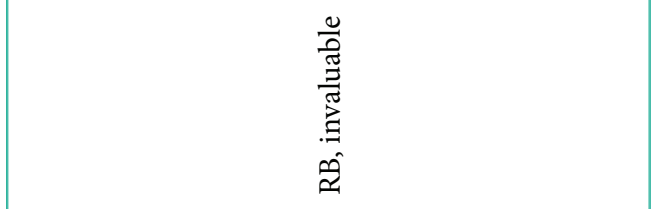 & શิ & 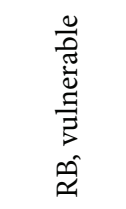 & 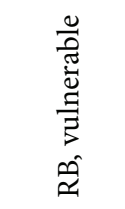 \\
\hline 山 & $\neg$ & $\mu$ & 山 \\
\hline 0 & 0 & $\exists$ & 0 \\
\hline$\simeq$ & $ـ$ & $\simeq$ & 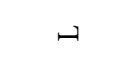 \\
\hline 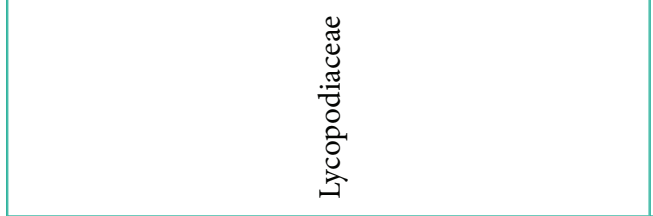 & 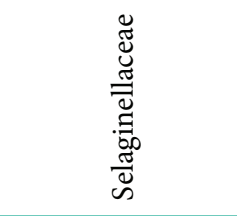 & 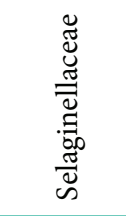 & 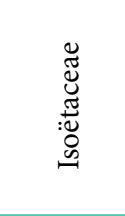 \\
\hline 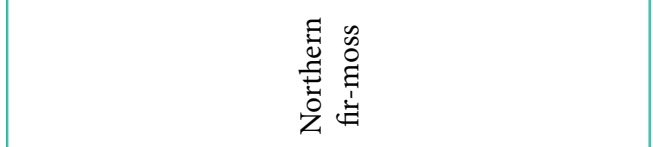 & 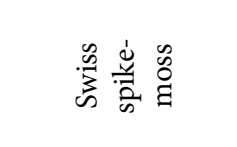 & 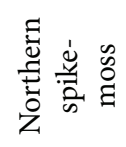 & 莺䓂 \\
\hline 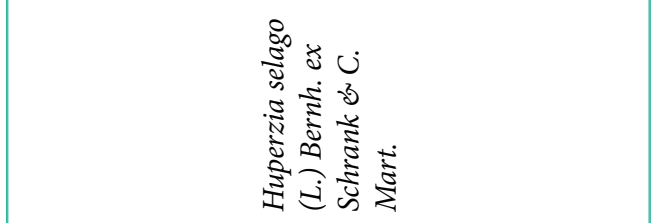 & 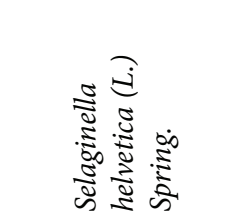 & 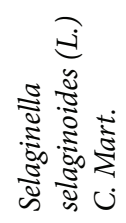 & 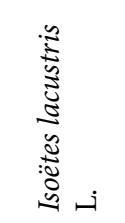 \\
\hline
\end{tabular}


After analyzing the State Register of Medicinal Products of Ukraine (14) we found out that most of the available drugs on the territory of Ukraine are manufactured and purchased abroad (Table 2).

In addition to the above-mentioned comprehensive homeopathic medications, there is also a well-known cosmetic product - a powder on the basis of dry extract of L. clavatum (double-acting powder), which has a calming effect, and helps to reduce irritation and redness. The country of origin of the product is Israel.

Table 2. Medications containing Lycopodium represented in the State Register of Medicines, Ukraine

\begin{tabular}{|c|c|c|c|}
\hline Name of the Drug & Active Substance & Producer & Applicant \\
\hline $\begin{array}{l}\text { METRO-ADNEX- } \\
\text { INJEEL }^{\oplus}\end{array}$ & $\begin{array}{l}\text { Lycopodium } \\
\text { clavatum }\end{array}$ & $\begin{array}{l}\text { Biologische Heilmittel Heel } \\
\text { GmbH., Germany }\end{array}$ & $\begin{array}{l}\text { Biologische Heilmittel Heel } \\
\text { GmbH., Germany }\end{array}$ \\
\hline $\begin{array}{l}\text { NUX VOMICA- } \\
\text { HOMACCORD }\end{array}$ & $\begin{array}{l}\text { Lycopodium } \\
\text { clavatum }\end{array}$ & $\begin{array}{l}\text { Biologische Heilmittel Heel } \\
\text { GmbH., Germany }\end{array}$ & $\begin{array}{l}\text { Biologische Heilmittel Heel } \\
\text { GmbH., Germany }\end{array}$ \\
\hline HEPEEL $^{\oplus} \mathrm{N}$ & $\begin{array}{l}\text { Lycopodium } \\
\text { clavatum }\end{array}$ & $\begin{array}{l}\text { Biologische Heilmittel Heel } \\
\text { GmbH., Germany }\end{array}$ & $\begin{array}{l}\text { Biologische Heilmittel Heel } \\
\text { GmbH., Germany }\end{array}$ \\
\hline HEPEEL $^{\oplus}$ & $\begin{array}{l}\text { Lycopodium } \\
\text { clavatum }\end{array}$ & $\begin{array}{l}\text { Biologische Heilmittel Heel } \\
\text { GmbH., Germany }\end{array}$ & $\begin{array}{l}\text { Biologische Heilmittel Heel } \\
\text { GmbH., Germany }\end{array}$ \\
\hline $\begin{array}{l}\text { PEKANA } \\
\text { NATURHEILMITTEL. }\end{array}$ & $\begin{array}{l}\text { Lycopodium } \\
\text { clavatum }\end{array}$ & $\begin{array}{l}\text { Biologische Heilmittel Heel } \\
\text { GmbH., Germany }\end{array}$ & $\begin{array}{l}\text { Biologische Heilmittel Heel } \\
\text { GmbH., Germany }\end{array}$ \\
\hline $\begin{array}{l}\text { MOMORDICA } \\
\text { COMPOSITUM }\end{array}$ & $\begin{array}{l}\text { Lycopodium } \\
\text { clavatum }\end{array}$ & $\begin{array}{l}\text { Biologische Heilmittel Heel } \\
\text { GmbH., Germany }\end{array}$ & $\begin{array}{l}\text { Biologische Heilmittel Heel } \\
\text { GmbH., Germany }\end{array}$ \\
\hline $\begin{array}{l}\text { TESTIS } \\
\text { COMPOSITUM }\end{array}$ & $\begin{array}{l}\text { Lycopodium } \\
\text { clavatum }\end{array}$ & $\begin{array}{l}\text { Biologische Heilmittel Heel } \\
\text { GmbH., Germany }\end{array}$ & $\begin{array}{l}\text { Biologische Heilmittel Heel } \\
\text { GmbH., Germany }\end{array}$ \\
\hline WEIGHT NORM & $\begin{array}{l}\text { Lycopodium } \\
\text { clavatum }\end{array}$ & $\begin{array}{l}\text { PC "National Homeopathic } \\
\text { Union", Ukraine }\end{array}$ & $\begin{array}{l}\text { PC "National Homeopathic } \\
\text { Union", Ukraine }\end{array}$ \\
\hline HOLE-GRAN & Lycopodium & $\begin{array}{l}\text { PC “National Homeopathic } \\
\text { Union”, Ukraine; } \\
\text { Ferrer Internacional, S.A., Spain }\end{array}$ & $\begin{array}{l}\text { PC “National Homeopathic } \\
\text { Union”, Ukraine; } \\
\text { Ferrer Internacional, S.A., Spain }\end{array}$ \\
\hline SCLERO-GRAN & $\begin{array}{l}\text { Lycopodium } \\
\text { clavatum }\end{array}$ & $\begin{array}{l}\text { PC "National Homeopathic } \\
\text { Union", Ukraine }\end{array}$ & $\begin{array}{l}\text { PC "National Homeopathic } \\
\text { Union", Ukraine }\end{array}$ \\
\hline $\begin{array}{l}\text { GRAPHITES } \\
\text { COSMOPLEX S } \\
\end{array}$ & $\begin{array}{l}\text { Lycopodium } \\
\text { clavatum }\end{array}$ & $\begin{array}{l}\text { Biologische Heilmittel Heel } \\
\text { GmbH., Germany }\end{array}$ & $\begin{array}{l}\text { Biologische Heilmittel Heel } \\
\text { GmbH., Germany }\end{array}$ \\
\hline ENERCEL- PLUS I. M & $\begin{array}{l}\text { Lycopodium } \\
\text { clavatum }\end{array}$ & Laboratories Vijosa S.A. de C.V. & Laboratories Vijosa S.A. de C.V. \\
\hline ENERCEL ${ }^{\oplus}$ FORTE & $\begin{array}{l}\text { Lycopodium } \\
\text { clavatum }\end{array}$ & Laboratories Vijosa S.A. de C.V. & Laboratories Vijosa S.A. de C.V. \\
\hline$\underline{\text { SOLVENCIUM }}$ & $\begin{array}{l}\text { Lycopodium } \\
\text { clavatum }\end{array}$ & PC “Biolik" & $\begin{array}{l}\text { LLC «Ukrainian pharmaceutical } \\
\text { company» }\end{array}$ \\
\hline $\begin{array}{l}\text { GEPATIUS “SPAG” } \\
\text { PEKANA }\end{array}$ & $\begin{array}{l}\text { Lycopodium } \\
\text { clavatum }\end{array}$ & $\begin{array}{l}\text { Pecana Heilmittel Heel GmbH., } \\
\text { Germany }\end{array}$ & $\begin{array}{l}\text { Pecana Heilmittel Heel GmbH., } \\
\text { Germany }\end{array}$ \\
\hline
\end{tabular}

${ }^{*}$ Drugs are in bold and underlined - the term of registration has expired. 
Medication containing Lycopods is also used in veterinary medicine. For example, Liarsin, which includes L. clavatum, is a complex homeopathic product, which has a corrective effect on protein, hydrocarbon, metabolism, restores impaired gastrointestinal function, stimulates the body's immune system. Its country of origin is Russia.

However, it should be noted that Lycopods have poisonous properties and thier use should be carried out under medical supervision.

It is likely that this situation with the research and use of Lycopods is primarily conditioned by the absence of a raw material base in Ukraine and their conservation status.

\section{CONCLUSION}

The results obtained from the use of Lycopodiophyta indicate that there is no medical or any other economic importance in Ukraine. Most species of Lycopods are distributed only in certain regions and have a conservation status. The populations of the studied species are few and sensitive to anthropogenic factors. Most homeopathic preparations containing Lycopods are produced in other countries, which is caused by a number of factors listed above.

\section{REFERENCES}

1. Grodzinsky AM, editor. Medicinal plants. Kyiv: Encyclopedic Directory; 1992.

2. Kharchenko NS, Karamyshev AN, Sula VI, Volodarsky LI. Medicinal plants and their use. Kyiv: Zdorov'ia; 1981.

3. Lin CC, Kan WS. Medicinal plants used for the treatment of hepatitis in Taiwan. Am J Chinese Med. 1990; 18(1-2):35-43. doi: 10.1142/ S0192415X9000006X.

4. Minarchenko VM. Medical vascular plants of Ukraine (medical and resource value). Kyiv: Fitosociocentre; 2005.

5. Nosal MA, Nosal IM. Medicinal plants and methods of their application in the people. Kyiv; 2013.

6. Grushvitsky IV, Zhilina SG, editors. Life of plants. V. 4. Mosses. Club mosses. Horsetails. Ferns. Gymnospermous plants. Moscow: Prosveshchenye; 1978.

7. Mosyakin SL, Fedoronchuk MM. Vascular plants of Ukraine: a nomenclatural checklist. Kiev; 1999.
8. Mosyakin SL, Tyshenko OV. A pragmatic phylogenetic classification of vascular Cryptogamic plants of the flora of Ukraine. Ukr Botan J. 2010; 67(6): 802-17.

9. Mukolaychuk VG. Botany. V. II. Course of lectures. Nikolaev: Nikolaev National Agrarian University; 2017.

10. Didukh YP, editor. Ecoflora of Ukraine. V. I. Kyiv: Fitosociocentre; 2000.

11. Hassan HM, Jiang ZH, Syed TA, Qin W. Northern Ontario medicinal plants. Can J Plant Sci. 2012; 92(5):815-28. doi:10.4141/cjps2012-006.

12. Prokydin JD, editor. The determinant of higher plants of Ukraine. Kyiv: Naukova dymka; 1987.

13. Didukh YP, editor. Red Book of Ukraine. Kyiv: Globalkonsaltyng; 2009.

14. State Register of Medicinal Products of Ukraine, Available at: http://www.drlz.com.ua/

15. Minarchenko VM, Tymchenko IA. Atlas of medicinal plants of Ukraine Kyiv: Fitosociocentre; 2002.

16. Tsvelev NN, editor. Conspectus Florae Europae Orientalis. Tomus 1. Petropoli-Mosqua: Consociatio editionum scientificarum KMK; 2012.

17. Allen D, Bilz M, Leaman DJ, Miller RM, Timoshyna A, Window J. European Red List of Medicinal Plants. Luxembourg: Publications Office of the European Union; 2014.

18. Bilz M, Kell SP, Maxted N, Lansdown RV. European Red List of Vascular Plants. Luxembourg: Publications Office of the European Union; 2011.

19. IUCN. Red List categories and criteria: Version 3.1. 2nd ed. Gland (Switzerland); Cambridge (UK): IUCN; 2012.

20. Ayer W. The Lycopodium alkaloids. Nat Prod Rep. 1991; 8(5):455-63. doi: 10.1039/np9910800455.

21. Czapski GA, Szypuła W, Kudlik M, Wileńska B, Kania M, Witold Danikiewicz W, et al. Assessment of antioxidative activity of alkaloids from $\mathrm{Hu}$ perzia selago and Diphasiastrum complanatum using in vitro systems. Folia Neuropathol. 2014; 52(4):394-406. doi: 10.5114/fn.2014.47840.

22. Czeczuga B. Carotenoids in sixty-six representatives of the Pteridophyta. Biochem Syst Ecol. 1985b; 13(3):221-30. doi:10.1016/0305-1978(85)90030-4.

23. Katakawa K, Mito H, Kogure N, Kitajima M, Wongseripipatana S, Arisawa M, et al. Ten new fawcettimine-related alkaloids from three species 
Tetyana Dvirna, Valentyna Minarchenko, Iryna Tymchenko

of Lycopodium. Tetrahedron. 2011; 67(35):6561-7. doi:10.1016/j.tet.2011.05.107.

24. Ma X, Gang DR. The Lycopodium alkaloids. Nat Prod Rep. 2004; 21(6):752-72. doi: 10.1039/ b409720n.

25. Filho JMB, Alencar AA, Nunes XP, de Andrade Tomaz AC, Sena-Filho JG, Athayde-Filho PF, et al. Sources of alpha-, beta-, gamma-, delta- and epsilon-carotenes: A twentieth century review. Braz J Pharmacogn. 2008; 18(1):135-54. doi:10.1590/ S0102-695X2008000100023.

26. Flora of North America Editorial Committee, editors. Flora of North America North of Mexico. Vol.2. New York \& Oxford: Oxford Univ Press; 1993. Available at: http://www.efloras.org/volume_ page.aspx?volume_id=1002\&flora_id=1

27. Halldorsdottir ES, Palmadottir RH, Nyberg NT, Olafsdottir ES. Phytochemical analysis of alkaloids from the Icelandic club moss Diphasiastrum alpinum (L.) Holub. Planta Med. 2012; 78(11):402. doi: 10.1055/s-0032-1321089.

28. Ishiuchi $K$, Kubota $T$, Ishiyama $H$, Hayashi $S$, Shibata T, Mori K, et al. Lyconadins D and E, and complanadine E, new Lycopodium alkaloids from Lycopodium complanatum. Bioorg Med Chem. 2011;19(2):749-53. doi: 10.1016/j.bmc.2010.12.025.

29. Ma X, Tan Ch, Zhu D, Gang DR, Xiao P. Huperzine A from Huperzia species - An ethnopharmacolgical review. J Ethnopharmacol. 2007; 113(1):15-34. doi: 10.1016/j.jep.2007.05.030.

30. Oirin B, Jay M. Etude chimiosystematique des Lycopodiales, Isoetales Selaginellales et Psilotales. Biochem Syst Ecol. 1978b; 6(2):99-102. doi:10.1016/0305-1978(78)90032-7.
31. Harborne JB, Baxter H, Moss GP, editors. Phytochemical Dictionary. A Handbook of Bioactive Compounds from Plants. 2nd Edition. London; Philadelphia: Taylor\&Francis; 1999.

32. Sokolov VS. Alkaloidonic plants of the USSR. AN USSR; 1952.

33. Voirin B. Distribution des composes polyphenoliques chez les Lycopodinees. Phytochemistry. 1972; 11(1):257-62. doi:10.1016/S0031-9422(00)90000-1.

34. Voirin BJM, Hauteville M. Isoetine, nouvelle flavone isoleé de Isoetes delilei et de Isoetes durieui. Phytochemistry. 1975; 14(1):257-60. doi:10.1016/0031-9422(75)85050-3.

35. Voirin BJM, Hauteville M. Selagine, nouvelle flavone isoleé de Huperzia selago. Phytochemistry. 1976; 15:840-1.

36. Voirin BJM. Apport de la Biochimie Flavonique à la systematique du genre Lycopodium. Biochem Syst Ecol. 1978a; 6(2):95-7. doi:10.1016/0305-1978(78)90031-5.

37. Voirin B. Recherches chimiques, taxinomiques etphysiologiques surlesflavonoides des Pteridophytes. Thèse. Docteur-Science. L’Université de Lyon; 1970.

38. Tsuda Y, Fujimoto T, Isobe K, Sano T, Kobayashi M. Chemotaxonomical studies on the triterpenoids of Lycopodium plants. Yakugaku Zasshi. 1974; 94(8):970-90. 